\title{
Aportaciones al CONOCIMIENTO TURÍSTICO DEL DESTINO Puerto Vallarta y su zona de influencia, desde el posgrado EN CIENCIAS PARA EL DESARROLLO, LA SUSTENTABILIDAD Y EL TURISMO
}

\author{
Carlos Gauna Ruiz de León \\ carlosg@cuc.udg.mx \\ Carlos R. Virgen Aguilar \\ rogeliov@cuc.udg.mx
}

Centro Universitario de la Costa, Universidad de Guadalajara

Se realiza un análisis del destino Puerto Vallarta y su zona de influencia, a través de los trabajos de tesis de posgrado (maestría y doctorado) en Ciencias para el desarrollo, la sustentabilidad y el turismo de la Universidad de Guadalajara, con una visión multidisciplinar, de acuerdo con temáticas propuestas desde la academia para el análisis de la actividad turística. Refiere la importancia que tiene el turismo en una de las regiones mexicanas con mayor desarrollo, que a su vez provoca impactos económicos, socioculturales y medioambientales; además, presenta aportaciones para la solución de los conflictos y el fortalecimiento de sus capacidades de manera sostenible.

Palabras clave: creación de conocimiento, Puerto Vallarta, turismo, desarrollo, sociedad, sustentabilidad.

\section{Contributions FROM the POSTGRADUATE PROGRAM IN SCIENCES FOR DEVELOPMENT, SUSTAINABILITY AND TOURISM TO KNOWLEDGE Creation regarding Puerto Vallarta and its area of influence}

An analysis of Puerto Vallarta's destination and its zone of influence is made through the theses of the University of Guadalajara's graduate program (doctoral and masters) in sciences for development, sustainability and tourism, with a multidisciplinary vision, according to the themes proposed by the academy for the analysis of tourism activity. It shows the importance of tourism in one of the mexican regions with the greatest development, which in turn causes economic, socio-cultural and environmental impacts. It also presents contributions for the solution of conflicts and the strengthening of its capacities in a sustainable manner.

Keywords: knowledge creation, Puerto Vallarta, tourism, development, society, sustainability. 


\section{Introducción}

—n la actualidad, la investigación y la formación de investigadores es un reto de las - universidades. Los recursos siempre son escasos y deben ser usados de forma adecuada hacia cumplir con los fines para los que fueron constituidas las instituciones de educación superior. Entre estos fines está la investigación científica, teniendo como objetivo la realización de trabajos pertinentes y de calidad. Un esfuerzo igual de importante es construir esquemas de educación superior de posgrado que formen a los nuevos investigadores. Esto es tan válido para las ciencias exactas como las ciencias sociales.

La construcción de un posgrado de calidad que atienda la problemática social asociada al turismo, ha sido la función de un grupo amplio de investigadores de diversas disciplinas que han aportado sus esfuerzos desde el Centro Universitario de la Costa de la Universidad de Guadalajara (CUCosta), con el apoyo de muchos otros investigadores, nacionales y extranjeros que se han sumado como asesores del proyecto de posgrado y como apoyo disciplinar de muchas de las tesis de nivel maestría y doctorado. Los resultados han sido valiosos. En los casi 20 años de esta experiencia se tienen egresados que ahora son investigadores en instituciones de educación superior, trabajando en la formación de profesionistas del futuro y haciendo investigación de calidad en busca de dar soluciones a los problemas que enfrenta la sociedad. Como parte de esta búsqueda, un objeto de estudio reiterado en las tesis de posgrado ha sido la actividad turística en la costa de Jalisco, por lo que se considera valioso recuperar los aportes realizados a la fecha. Así, este trabajo se aborda de forma multidisciplinar, a partir del conocimiento generado desde el Posgrado en Ciencias para el Desarrollo, la Sustentabilidad y el Turismo.

El objetivo es analizar las condiciones que prevalecen en la actividad turística en uno de los territorios más importantes del país, como es Puerto Vallarta y su área de influencia, explicando su conformación histórica, las formas en que se ha impulsado el proceso de desarrollo, con todas sus virtudes y también con sus defectos, la dinámica social y su relación con el entorno, la forma como se ha ocupado el espacio y los conflictos que se provocan, a través de la visión de los trabajos de los alumnos del posgrado que, con el rigor académico manifestado, se puede explicar de manera integral la realidad social, económica, cultural y medioambiental.

El destino Puerto Vallarta se integra, para los fines de este trabajo, por la zona conurbada de Puerto Vallarta, Jalisco - Bahía de Banderas, Nayarit, y la zona de influencia de la Costalegre, Jalisco, que la conforman los municipios de Cihuatlán, La Huerta, Tomatlán y Cabo Corrientes. 


\section{La formación de investigadores y la investigación en turismo}

La formación de investigadores desde los estudios de posgrado es relevante por su trascendencia en la actividad académica, esto es aún mayor cuando este trabajo se realiza en un país con una baja producción de investigación y es prioridad cuando se trata de una temática compleja como el turismo, que requiere una atención multidisciplinar.

Existen estudios que analizan la calidad de la formación de los investigadores, particularmente sobre las políticas públicas en México que se generan desde el gobierno federal a partir de la Secretaría de Educación Pública (SEP) y del Consejo Nacional de Ciencia y Tecnología (Conacyt), haciendo una valoración crítica sobre los impactos y resultados obtenidos y presentando propuestas para su mejora (Rivas, 2004; Hernández y Nieto, 2010; Ortiz-Villanueva, 2013; Abreu-Hernández y Cruz-Flores, 2015); de forma particular el libro La calidad del pregrado y el posgrado, una mirada latinoamericana (Marum y Rosario, 2015), que es resultado de una conferencia latinoamericana sobre educación superior. En la misma temática existe un trabajo que da cuenta del impacto del posgrado en Argentina (Barky y Dávila, 2016).

De forma particular se tiene un trabajo sobre el impacto del posgrado en la Universidad Autónoma de Nayarit (Becerra, 2017) y dos documentos tratan especialmente sobre la formación de doctores en educación (Pacheco, 2011; Zaldívar, Canto y Quintero, 2018). Otras investigaciones analizan las características de los estudiantes de posgrado, la calidad de su formación, el proceso de egreso y el impacto en la sociedad (Contreras y Urrutia, 2017) y cómo se enfrentan los egresados al mundo del empleo como investigadores (Chavoya, 2013).

En cuanto a la investigación en turismo, se tienen trabajos que tratan sobre la problemática en México (López y Palomino, 2007; Osorio, Castillo y Moreno, 2008; Pimentel, 2016; Arnaiz, Pimentel y Gauna, 2017; Gómez-Nieves, 2018). En Uruguay, Pimentel y Carvalho (2019); en Ecuador, Pimentel, Costa y Bifano (2017); en Colombia, Castillo-Palacios y Zuñiga-Collazos (2015), en especial el análisis de la educación superior en turismo en América Latina y España, que recibió el premio "FITUR 2017" (Celis, 2019) y un análisis crítico sobre la formación en turismo en los nuevos tiempos (César y César, 2016).

En Brasil se ha realizado un estudio particular sobre la relevancia del turismo como tema de investigación de los posgrados en geografía (Silva y Fransualdo, 2019) y otro sobre la difusión de la investigación turística (Picazo, Moreno y León, 2012). 
Cabe destacar los trabajos que se han realizado desde la Academia Mexicana de Investigación Turística (AMIT) sobre la situación que guarda la investigación turística en México (Osorio y Korstanje, 2017; Monterrubio, 2017; Jiménez y Velázquez, 2017; Hiernaux, 2018), sobre un análisis de la producción académica de los investigadores del turismo en México (Rodríguez y Vargas, 2018) y la evaluación de la política científica de la investigación turística en México (Bringas y Verduzco, 2019).

El trabajo de formar investigadores en México tiene su soporte en el Programa Nacional de Posgrados de Calidad (PNPC) del Consejo Nacional de Ciencia y Tecnología del gobierno de la República (Conacyt, 2019), el cual funciona de manera ininterrumpida desde 1991. Para el ingreso y la promoción al PNPC es necesario que el posgrado solicite de manera voluntaria la evaluación, la cual se basa en criterios objetivos en diversas secciones que integran el trabajo académico de un posgrado de calidad (Estudiantes, Núcleo académico básico, Plan de estudios, Movilidad, Productividad académica).

Tabla 1. Posgrados en el Programa Nacional de Posgrados de Calidad de Conacyt

\begin{tabular}{|l|c|c|c|c|}
\hline \multicolumn{1}{|c|}{ Tipo } & Doctorado & Maestría & Especialidad & Total \\
\hline Competencia internacional & 89 & 132 & 24 & 245 \\
\hline Consolidado & 189 & 372 & 101 & 662 \\
\hline En desarrollo & 209 & 503 & 209 & 921 \\
\hline De nueva creación & 171 & 228 & 69 & 468 \\
\hline Total & 658 & 1235 & 403 & 2296 \\
\hline
\end{tabular}

Fuente: Elaboración propia a partir de información del portal web del Conacyt (2019).

En el año 2003 se conformó el proyecto de posgrado en "Ciencias para el desarrollo sustentable" (maestría y doctorado) el cual estaba dirigido a formar expertos que atendieran los problemas sociales de acuerdo a las Líneas de generación y aplicación del conocimiento (LGAC) de "Desarrollo local y desarrollo regional", "Desarrollo turístico sostenible" y "Sociedades en transición", basadas en los objetivos que se desarrollaban en el Centro de investigación para el desarrollo turístico sostenible (Cedestur), logrando para ambos niveles el reconocimiento dentro del PNPC.

En el año 2012, por cuestiones administrativas se tuvo que cerrar la primera estructura del posgrado, rehaciendo el modelo, pero manteniendo la parte esencial que es el núcleo académico, generando modificaciones que se consideraron adecuadas a partir de la experiencia. Se puede considerar de forma administrativa que existen dos épocas del posgrado, durante la segunda, se le denominó: Ciencias para el Desarrollo, la Sustentabilidad y el Turismo (PCDST) (Gauna y Vargas, 2017). En este periodo se 
logró que ambos posgrados obtuvieran un reconocimiento dentro del PNPC: la maestría ha avanzado al nivel de "en desarrollo" y el doctorado está en el nivel de "nueva creación” y durante el año 2020 será nuevamente evaluado.

La fundamentación teórica del posgrado se basa en el desarrollo y los procesos de planeación para alcanzarlo de forma particular desde la visión más amplia que ofrece la sustentabilidad. El turismo se considera como un modelo de desarrollo, pues a partir de esta actividad se pueden lograr los objetivos del desarrollo en los lugares que se lleve a cabo, siempre que genere beneficios reales a la sociedad y el uso del espacio lo realice cuidando el medio natural (CEED-Cedestur, 2001, p. 10).

Así, el turismo es más que una actividad, se puede considerar como un modelo de desarrollo que requiere un proceso de planeación, que se integra en los territorios y que concibe a la sociedad como un elemento fundamental. No se puede realizar ni pensar solo en los turistas y en los elementos inmobiliarios que se requieren en su operación:

"El turismo exige en su proceso de planificación para el desarrollo de un tratamiento interdisciplinario. El enfoque parcial de su desarrollo es precisamente el que ha dado lugar [...] a los efectos negativos que tanto se han señalado del turismo y la falta de un análisis sistemático ha impedido que el mismo sea evaluado y comparado en una forma justa [...] que pudiera coadyuvar al desarrollo económico y social de los países y regiones donde se genera" (Virgen, 2014, p.74).

\section{Metodología}

El esfuerzo de dar a conocer las aportaciones realizadas desde el posgrado, que muestran la calidad y pertinencia del proyecto, permite construir un relato que puede explicar de una manera amplia y profunda las condiciones de un destino turístico desde diversas disciplinas, lo que hace más enriquecedor el proceso, sobre todo cuando el destino es un referente de calidad e icónico dentro del turismo mexicano, como lo es Puerto Vallarta.

Los documentos analizados están resguardados en la biblioteca central del CUCosta. En la primera fase se ordenaron las tesis para ambos niveles del posgrado. Esta tarea se realizó gracias al apoyo de estudiantes del programa Delfín, en el verano de 2019. Se contrastó con la información de las bases de datos administrativas de la Universidad de Guadalajara para identificar a todos aquellos estudiantes que estuvieran graduados. 
Posteriormente, se vació la información relevante de las tesis en una base de datos: nombre del sustentante, director, nombre de la tesis, metodología aplicada, principales aportaciones, espacio temporal, espacio físico donde se realizó el trabajo de tesis y de observaciones en su lectura que fueran importantes. Para cada uno de los trabajos se determinó la LGAC a la que están adscritos en el posgrado. La concordancia de los trabajos de tesis con la teoría en la que se planeó el posgrado permite identificar que se han alcanzado los objetivos académicos planteados.

Toda vez que el turismo no se encuentra dentro de la clasificación disciplinar generada por el Conacyt, se determinó hacer un conteo de proyectos de tesis que abordan esta temática, Esto se sustenta en el perfil del posgrado y de las problemáticas asociadas a dicho tema en la región de la costa del Pacífico medio mexicano. Con la información de cada trabajo, se procedió a realizar un análisis descriptivo a partir de tablas estadísticas que dan cuenta del proceso de formación en el posgrado.

Teniendo presente el objetivo de esta indagación, se seleccionaron 69 trabajos que cumplen con los criterios de tener relación con el área de estudio y que se ajustan a la propuesta de distribución temática de la investigación turística propuesta por Osorio, Castillo y Moreno (2008).

Una vez concluido este trabajo se presenta una discusión donde se reflexiona sobre las aportaciones teóricas y metodológicas. También incluye un apartado de conclusiones sobre las aportaciones del programa de posgrado en beneficio de la sociedad del destino.

\section{Resultados del programa de posgrado}

De acuerdo con el reporte técnico de la formación de investigadores del PCDST, desde el año 2005 se han presentado 70 tesis de maestría y desde el 2006 para el doctorado se han presentado 67 (Tabla 2). Actualmente están matriculados o han concluido sus créditos curriculares otros 48 alumnos en el doctorado y 47 alumnos de la maestría, lo que augura que este número de trabajos de tesis seguirá creciendo de forma regular.

Tabla 2. Número de tesis por generación y nivel de posgrado

\begin{tabular}{|c|c|c|}
\hline Época & Doctorado & Maestría \\
\hline Primera & $\mathbf{5 5}$ & $\mathbf{4 6}$ \\
\hline Segunda & 12 & 24 \\
\hline Total & $\mathbf{6 7}$ & $\mathbf{7 0}$ \\
\hline
\end{tabular}

Fuente: Reporte técnico de la formación de investigadores en el PCDST (2020). 
Un dato significativo es que $49 \%$ de los graduados son hombres y $51 \%$ son mujeres, manteniendo esta igualdad en los dos niveles y en las dos épocas del posgrado (Tabla 3).

Tabla 3. Sustentantes del posgrado por nivel y sexo

\begin{tabular}{|c|c|c|c|c|c|c|}
\hline & \multicolumn{3}{c|}{ Doctorado } & \multicolumn{3}{c|}{ Maestría } \\
\hline Masculino & Primera & Segunda & Total & Primera & Segunda & Total \\
\hline Femenino & 28 & 5 & 33 & 24 & 10 & 34 \\
\hline Total & 27 & 7 & 34 & 22 & 14 & 36 \\
\hline
\end{tabular}

Fuente: Reporte técnico de la formación de investigadores en el PCDST (2020).

De los alumnos titulados del posgrado, 19 del doctorado (el 35.8\%) y 8 de la maestría (11.4\%) lograron ingresar al Sistema Nacional de Investigadores (SNI) de Conacyt. A la fecha, 45 profesores han fungido como directores de tesis y solo dos académicos de todos ellos no cuentan con el nivel de doctorado en la actualidad, pero sí tienen el nivel de maestría.

De acuerdo con la revisión de los trabajos adscritos a las LGAC del posgrado (Tabla 4), se tiene que en "Desarrollo turístico sustentable", se han generado 32 tesis de doctorado y 43 de la maestría; en "Desarrollo local y desarrollo regional" se cuenta con 16 tesis de doctorado y 18 de maestría, y en "Sociedades en transición" hay 19 tesis de doctorado y 9 de maestría. Muchos de estos trabajos, aunque se clasificaron en alguna de las tres líneas, tienen elementos de análisis que intervienen en las otras dos.

Respecto a la ubicación física de las investigaciones (Tabla 5), existen trabajos de Brasil, Colombia y Argentina en el doctorado, y un trabajo de España presentado en la maestría. A nivel nacional existen tesis de 8 estados: de Jalisco se realizaron 68 trabajos de los cuales 54 corresponden a Puerto Vallarta y 8 a la región Costalegre; otros 12 trabajos tienen una dimensión regional de la Bahía de Banderas. Del vecino estado de Nayarit, se realizaron 27 trabajos, de los municipios de Bahía de Banderas, Tepic y Compostela, y uno a nivel estatal. Adicionalmente hay trabajos de los estados de Baja California Sur, Sonora, Sinaloa, Chiapas, Oaxaca, Quintana Roo y Michoacán. 
Tabla 4. Distribución de los trabajos de acuerdo con las LGAC del Posgrado (por grado y época).

\begin{tabular}{|c|c|c|c|c|c|c|c|}
\hline \multirow{2}{*}{ LGAC } & \multicolumn{3}{|c|}{ Doctorado } & \multicolumn{3}{c|}{ Maestría } & \multirow{2}{*}{ Total } \\
\cline { 2 - 9 } & Primera & Segunda & Subtotal & Primera & Segunda & Subtotal & \\
\hline Desarrollo local y Desarrollo regional & 12 & 4 & 16 & 11 & 7 & 18 & 34 \\
\hline Desarrollo turístico sustentable & 26 & 6 & 32 & 33 & 10 & 43 & 75 \\
\hline Sociedades en transición & 17 & 2 & 19 & 2 & 7 & 9 & 28 \\
\hline Total & 55 & 12 & 67 & 46 & 24 & 70 & 137 \\
\hline
\end{tabular}

Fuente: Reporte técnico de la formación de investigadores en el PCDST (2020).

Es importante mencionar que 13 trabajos no tienen ubicación geográfica: 12 de ellos de doctorado, por ser investigaciones de procesos educativos, y un trabajo de maestría que es un análisis teórico sobre calidad de vida.

Tabla 5. Identificación del área de estudio de las tesis según nivel de posgrado

\begin{tabular}{|c|c|c|c|c|c|c|c|}
\hline $\begin{array}{c}\text { Cobertura espacial } \\
\text { de las tesis }\end{array}$ & Doctorado & Maestría & Total & $\begin{array}{c}\text { Cobertura espacial } \\
\text { de las tesis }\end{array}$ & Doctorado & Maestría & Total \\
\hline \multicolumn{4}{|c|}{ País } & \multicolumn{4}{|c|}{ Local: Jalisco } \\
\hline Argentina & 1 & & 1 & Puerto Vallarta & 17 & 37 & 54 \\
\hline Brasil & 1 & & 1 & Cabo Corrientes & 1 & 1 & 2 \\
\hline Colombia & 2 & & 2 & Concepción de Buenos Aires & 1 & & 1 \\
\hline España & & 1 & 1 & Ejutla & & 1 & 1 \\
\hline \multicolumn{4}{|c|}{ Nacional } & Talpa de Allende & & 1 & 1 \\
\hline México & 2 & & 2 & Zapopan, Jalisco & & 1 & 1 \\
\hline \multicolumn{4}{|c|}{ Estatal } & Puerto Vallarta/Tomatlán & & 1 & 1 \\
\hline Baja California Sur & 1 & & 1 & Costalegre & 3 & 5 & 8 \\
\hline Chiapas & 1 & & 1 & \multicolumn{4}{|c|}{ Local: Nayarit } \\
\hline Michoacán & 1 & & 1 & Bahía de Banderas & 8 & 12 & 20 \\
\hline Nayarit & 1 & & 1 & Compostela & 1 & & 1 \\
\hline Sinaloa & 3 & & 3 & Tepic & 5 & & 5 \\
\hline Sonora & 1 & & 1 & \multirow{2}{*}{ Reg. de Bahía de Banderas } & \multirow{2}{*}{5} & \multirow{2}{*}{7} & \multirow{2}{*}{12} \\
\hline Quintana Roo & & 1 & 1 & & & & \\
\hline
\end{tabular}

Fuente: Reporte técnico de la formación de investigadores en el PCDST (2020) y distribución conceptual del turismo. 


\section{El destino Puerto Vallarta y su zona de influencia a través de los estudios de tesis del posgrado}

Puerto Vallarta es el segundo destino turístico de playa del país y el primero del Pacífico Mexicano. Recibe más de seis millones de visitantes al año (tabla 6), que disfrutan las bellezas naturales del excelente clima casi todo el año y una atención esmerada por sus habitantes.

Tabla 6. Turistas hospedados según localidad 2018

\begin{tabular}{|c|c|c|c|}
\hline Localidad & Nacionales & Extranjeros & Total \\
\hline Nuevo Vallarta, Nayarit & 796912 & 2006526 & 2803438 \\
\hline Puerto Vallarta, Jalisco & 1368354 & 1963646 & 3331990 \\
\hline Costalegre, Jalisco & 77765 & 18518 & 96283 \\
\hline Total & 2243031 & 3988680 & 6231711 \\
\hline
\end{tabular}

Fuente: Datatur-Sectur (2018).

En la región vive más de medio millón de habitantes, tomando en consideración que para el año 2010, de acuerdo con los datos del Instituto Nacional de Estadística y Geografía, INEGI (2010), el municipio de Puerto Vallarta, Jalisco tenía 255 mil 681 habitantes. Los municipios que conforman la Costalegre, Jalisco, tenían 107527 habitantes y el municipio de Bahía de Banderas, Nayarit, tenía 124209 habitantes, lo que nos daba para 2010 cerca de medio millón de habitantes. Tomando en consideración el crecimiento demográfico promedio, se puede estimar que ahora viven en la región de estudio más de 700 mil habitantes (Virgen y Gauna, 2011).

En los últimos 50 años se ha generado un constante crecimiento de la actividad turística, creando empresas de diverso tamaño y ampliando la operación de un clúster que ha permitido el asentamiento de emprendimientos asociados. Estos han hecho crecer a las poblaciones de pequeñas localidades hasta convertirse en grandes centros urbanos que configuran un polo de desarrollo regional.

Para comprender la dinámica de la sociedad asentada en este territorio, sus retos y los procesos complejos que se han generado, existen trabajos de investigación que aportan a la comprensión del territorio y de la sociedad que allí vive (César y Arnaiz, 2005; Cornejo-Ortega, Chávez-Dagostino y Espinoza-Sánchez, 2019).

A partir de los 167 trabajos presentados en el posgrado, se seleccionaron 69 tesis, tanto de maestría como de doctorado (Tabla 7), que tratan de la temática del turismo en la zona de estudio, haciendo la pertinente aclaración que en algunos trabajos se puede considerar su relación con el turismo de forma indirecta, toda vez que 
tratan sobre el territorio y los habitantes de localidades que están relacionados por los impactos y las condiciones que la actividad turística genera.

Tabla 7. Distribución de los trabajos de tesis en función de la temática del turismo

\begin{tabular}{|c|c|c|c|}
\hline Tema & Subtema & Nivel & Tesis \\
\hline \multirow{3}{*}{$\begin{array}{l}\text { Legislación, gestión } \\
\text { y políticas públicas del } \\
\text { turismo }\end{array}$} & Políticas públicas & Maestría & Franco (2006). \\
\hline & \multirow{2}{*}{$\begin{array}{l}\text { Gestión de riesgos, } \\
\text { seguridad y preven- } \\
\text { ción social }\end{array}$} & Doctorado & Cabral (2006); Vargas (2013). \\
\hline & & Maestría & $\begin{array}{l}\text { Casas (2017); Gutiérrez (2018); Huerta (2018); } \\
\text { Pérez (2018). }\end{array}$ \\
\hline \multirow{2}{*}{$\begin{array}{l}\text { Turismo, cultura, patri- } \\
\text { monio } \\
\text { e identidad }\end{array}$} & \multirow{2}{*}{$\begin{array}{l}\text { Patrimonio y } \\
\text { gastronomía }\end{array}$} & Doctorado & $\begin{array}{l}\text { Ceballos (2009); P. Núñez (2008); Scartascini } \\
\text { (2009). }\end{array}$ \\
\hline & & Maestría & $\begin{array}{l}\text { González (2006); Cabrera (2008); De Niz (2018); } \\
\text { López S. (2006); Mora (2006). }\end{array}$ \\
\hline \multirow{3}{*}{$\begin{array}{l}\text { Estudios económicos } \\
\text { y administrativos del } \\
\text { turismo }\end{array}$} & \multirow{2}{*}{ Pymes turísticas } & Doctorado & García (2018); Vargas (2017) \\
\hline & & Maestría & Petroskova (2019). \\
\hline & $\begin{array}{l}\text { Tecnologías de la } \\
\text { Información (TICs) }\end{array}$ & Maestría & Cortés (2008). \\
\hline \multirow{3}{*}{$\begin{array}{l}\text { Mercados, segmentos } \\
\text { y desarrollo de nuevos } \\
\text { productos }\end{array}$} & \multirow{2}{*}{$\begin{array}{l}\text { Estudios de creación } \\
\text { y gestión de oferta y } \\
\text { productos turísticos }\end{array}$} & Doctorado & $\begin{array}{l}\text { Virgen (2007); Hernández (2016); Zepeda (2018); } \\
\text { Olivares (2008). }\end{array}$ \\
\hline & & Maestría & $\begin{array}{l}\text { César (2005); Navarro (2005); Huizar (2005); Zo- } \\
\text { rrilla (2006); Tiznado (2007); Calderón (2008); S. } \\
\text { Núñez (2008); Hernández, 2010; Santos (2017). }\end{array}$ \\
\hline & Estudios de demanda & Maestría & González (2010). \\
\hline \multirow{6}{*}{$\begin{array}{l}\text { Espacio, planeación, } \\
\text { sustentabilidad y } \\
\text { desarrollo local }\end{array}$} & \multirow[b]{2}{*}{$\begin{array}{l}\text { Ordenamiento, } \\
\text { planeación del } \\
\text { espacio }\end{array}$} & Doctorado & Fernández, 2009; Rendón, 2017; Dávalos (2019). \\
\hline & & Maestría & $\begin{array}{l}\text { Baños (2005); Calzada (2005); López R. (2006), } \\
\text { Tovar (2006); Chavoya (2006); Tapia (2006); Reyes } \\
\text { (2009); Molina (2017); Segura (2017); Jáuregui } \\
\text { (2018); García (2019); Lara (2019). }\end{array}$ \\
\hline & \multirow[b]{2}{*}{ Sustentabilidad } & Doctorado & Chávez (2007); Castro (2009); Zepeda (2019). \\
\hline & & Maestría & $\begin{array}{l}\text { Beets (2006); Hernández (2008); Kosonoy (2008); } \\
\text { Cruz (2009); Herrera (2019). }\end{array}$ \\
\hline & \multirow[b]{2}{*}{$\begin{array}{l}\text { Estudios de } \\
\text { comunidades locales }\end{array}$} & Doctorado & Fernández (2014). \\
\hline & & Maestría & $\begin{array}{l}\text { Sánchez (2005); Rueda (2005); Gómez (2006); } \\
\text { Bravo (2006); García (2008); Villaseñor (2010); } \\
\text { Noriega (2013); Llamas (2018); Ortiz (2017); Nájera } \\
\text { (2018); Gutiérrez R. (2018); Villanueva (2019). }\end{array}$ \\
\hline
\end{tabular}

Fuente: Reporte técnico de la formación de investigadores en el PCDST (2020) y distribución conceptual del turismo. 
En una revisión de los trabajos que se presentan, en el tema de legislación, gestión y políticas públicas del turismo así como en el subtema de políticas públicas, se tiene una propuesta de organización administrativa del área de turismo del ayuntamiento de Puerto Vallarta (Franco, 2006), que explica que no hay claridad sobre el nivel de su intervención en la actividad del turismo por los tres niveles de gobierno. Existen diversas posturas del tratamiento del turismo y en muchos ordenamientos no queda claro las responsabilidades y alcances que debería tener cada uno de ellos, por lo que se genera una propuesta para construir un marco legal que trate al turismo y permita hacer operativa la gestión desde el ayuntamiento, más allá de una actividad económica, y que se integren las preocupaciones sociales y medioambientales.

En el subtema de Gestión de riesgos, seguridad y prevención social, por el carácter multidisciplinar del posgrado, un grupo de psicólogos sociales y sociólogos se han enfocado en los impactos sociales que se provocan en la sociedad de Puerto Vallarta, particularmente asociados al turismo. Se basa en el impacto que se genera en la sociedad producto de los procesos de transición que se tienen por el crecimiento de la población y los efectos de transculturización y presión laboral. Hay dos tipos de trabajos, los que tratan los procesos de marginación en las zonas pauperizadas y de las condiciones de vida de las poblaciones asentadas en esas zonas (Cabral, 2006; Casas, 2017), y los trabajos que tratan de la presión psicosocial que tienen los trabajadores, de forma particular las mujeres, lo que provoca condiciones de inestabilidad social, familiar y laboral a partir de las condiciones de vida y laboral en los hoteles (Vargas, 2013; Huerta, 2018; Gutiérrez, 2018; Pérez, 2018). Todos estos trabajos han usado instrumentos estandarizados estadísticos para conocer la situación de los trabajadores de acuerdo con cada pregunta de investigación.

En el tema de Turismo, cultura, patrimonio e identidad, se tienen tesis en el subtema de Patrimonio y Gastronomía. Por la temática tan amplia se hace una subclasificación de los trabajos. Se analiza la relación de las religiones en la sociedad, por el impacto que tiene la trasformación de la sociedad en relación con la actividad turística, de forma particular de la población migrante en Bahía de Banderas y los cambios que se provocan en su identidad, especialmente en sus creencias religiosas, teniendo un incremento de grupos evangélicos contra un decremento en el catolicismo. La mayor parte de los trabajadores son migrantes y en sus comunidades de origen realizaban trabajos tradicionales, asociados al campo, con fuertes raíces culturales y religiosas que se modifican por su nuevo lugar de residencia y trabajo (Ceballos, 2009).

En cuanto al impacto de la gastronomía en las comunidades y el uso de cocinas tradicionales, se plantean las potencialidades de la actividad gastronómica asociada 
al turismo (Cabrera, 2008) y a las capacidades de generación de clúster en los restaurantes del destino (De Niz, 2018). Se concluye que existen limitados procesos de encadenamientos productivos y que la mayor parte de las empresas realizan sus compras de forma particular en los grandes centros comerciales.

Por un lado, se analiza la relación de la cultura con el destino, generando información sobre la importancia que reviste como un apoyo a la actividad turística y a la preservación de las tradiciones de las localidades (Mora, 2006). De forma particular destaca el tema de las artesanías, siendo un elemento que valoriza a la cultura y que es indispensable en el turismo, pues son representaciones culturales muy apreciadas por los visitantes (López S., 2006). Igualmente, se revisa el papel que juegan los guías de turistas como promotores de la cultura e identidad del destino, como elemento distintivo en la actividad turística de Puerto Vallarta (González, 2006). Se señala que se deben generar más y mejores mecanismos de capacitación y concientización para los guías de turistas y se requieren aplicar las normativas legales para evitar que este trabajo lo realicen personas no capacitadas.

Sobre el desarrollo histórico del destino se cuenta con dos abordajes: El primero plantea un análisis a partir de fuentes periodísticas y de documentos oficiales que explican el momento histórico de despegue de Puerto Vallarta entre los años $60 \mathrm{y}$ 70, apuntando la importancia que tuvo la inversión pública con los equipamientos y el financiamiento para el desarrollo, pero también la relevancia del impulso por los empresarios que creyeron en el proyecto (Núñez, 2008). En el segundo trabajo se hace un recuento histórico a través de la recuperación oral de los pobladores originales sobre cómo vivieron la trasformación de la localidad y el impacto que esto tuvo en la vida de los habitantes de Puerto Vallarta (Scartascini, 2009).

En los estudios económicos y administrativos del turismo, en el subtema de Pymes turísticas, se tiene una caracterización de las micro, pequeñas y medianas empresas, a partir de criterios de competitividad, con los agregados de sostenibles y de responsabilidad social corporativa como estrategias de desarrollo en las empresas hoteleras de Puerto Vallarta, aportando que se debe trabajar en la mejora de las capacidades de las empresas turísticas, de forma particular en los hoteles de tres y cuatro estrellas (García, 2018). Otro estudio presenta las características competitivas de las microempresas en San Francisco, una localidad turística del litoral de Nayarit (Petroskova, 2019). En estos trabajos se tienen resultados favorables sobre los hoteles, aunque no tan positivos en la localidad de Nayarit, debido a la incertidumbre en la inversión, los limitados procesos empresariales que se generan en la localidad, así como la falta de incentivos y apoyos desde el gobierno. 
En otra tesis se exploran las capacidades que tienen los empresarios de la región a partir de la propuesta metodológica diseñada por el "Global Entrepreneurship Monitor (GEM)" (Vargas, 2017), encontrando que existen mejores condiciones de emprendimiento en Puerto Vallarta que los que se tienen a nivel nacional, debido al dinamismo que se genera en la región a partir del turismo. Este trabajo se realizó a través de un instrumento estandarizado estadísticamente, aplicado a dueños y responsables de establecimientos de Puerto Vallarta.

Solo hay un trabajo que atiende la temática de las Tecnologías de la Información y la Comunicación (TIC) (Cortés, 2008). La investigación se realizó cuando empezaba el E-comercio y estaba en una fase primaria el uso de las tecnologías. Da cuenta del inicio de la transformación digital de las empresas locales y las transnacionales, que al generar mecanismos para incidir a través de procesos virtuales facilitó el acceso a nuevos mercados.

En la temática de Mercados, segmentos y desarrollo de nuevos productos, en la oferta turística y la creación de nuevos productos, se tienen varios apartados: en el que trata de estudios sobre la oferta hotelera, se ubican los estudios sobre la evolución de la industria hotelera en el destino, los procesos de certificación de las empresas turísticas y el ciclo de vida del producto. Estos trabajos dan cuenta de la importancia que tienen las empresas turísticas en el destino, cómo se han desarrollado en el tiempo y la relevancia que tiene la certificación para acceder a nuevos y mejores mercados (Zorrilla, 2006) (Calderón, 2008) (Núñez Martínez, S. 2008).

Es de resaltar el estudio comparativo de dos zonas turísticas: Careyes en la Costalegre, y Nuevo Vallarta en Bahía de Banderas, en el que se analizan las diferentes condiciones de dos modelos de desarrollo turísticos exitosos, los cuales tienen ofertas diferenciadas y se han convertido en iconos del turismo en la costa de Jalisco (César, 2005). Este trabajo recibió el Premio de Tesis de Maestría 2006 otorgado por la Secretaría de Turismo Federal.

En otra de las investigaciones, (Santos, 2017) realizó un modelo matemático para determinar el valor económico de la actividad hotelera mediante estudios probabilísticos, a partir de la información disponible. No obstante la limitación de los datos disponibles, el ejercicio permitió identificar una aproximación económica del valor que se genera en el sector hotelero en el destino con la información de hoteles de cinco estrellas.

Se analiza también la relación entre la empresa hotelera y las estructuras laborales, centrada en la atención sobre la absorción de mano de obra con poca calificación y en las condiciones desfavorables para los trabajadores debido a la flexibilidad labo- 
ral, situación que afecta no solo a los empleados, sino que repercute en la pérdida de competitividad de las empresas (Navarro, 2005; Hernández, 2010). Estos trabajos están asociados en sus conclusiones a los desarrollados en la temática social sobre las condiciones de vida de los trabajadores.

Sobre la construcción de la marca Puerto Vallarta, se explica su integración desde la idea de los imaginarios, lo que cada símbolo de la marca representa para el destino, cuyo principal mecanismo publicitario es el emblema de las características identitarias tanto culturales como naturales que le dan singularidad y que le han permitido tener un lugar bien ganado en los mercados turísticos nacionales e internacionales (Hernández, 2016; Zepeda , 2018).

A partir de la imagen proyectada del destino Puerto Vallarta, se analiza la necesidad de generar nuevos productos turísticos que renueven al destino por la enorme competencia de los mercados del turismo (Olivares, 2008), así como la diversificación de la oferta turística de segundas residencias (Huizar, 2005). En el mismo sentido, se reflexiona sobre la relevancia del segmento de turismo de cruceros y la necesidad de que sea una actividad sustentable (Tiznado, 2007), situación que es prioritaria por la transformación del puerto de cruceros generada en Puerto Vallarta.

Un trabajo que analiza al destino de una manera amplia es el estudio del ciclo de vida del destino, llegando a la conclusión que Puerto Vallarta está en un nivel de madurez que requiere una reactivación para generar nuevas condiciones de crecimiento de la demanda. Las limitantes de este trabajo se deben a que solo toma como base los datos de la ciudad de Puerto Vallarta sin incluir a la zona de Nuevo Vallarta, Nayarit, pero por aproximación se expresa que la situación no es diferente con su inclusión. Lo más valioso de este trabajo es que a partir de una metodología reconocida en el mundo del turismo se analiza la situación histórica del destino (Virgen, 2007).

Existe un trabajo que analiza la satisfacción de los turistas a partir de la imagen proyectada del destino. En él se explora, a través de estudios de satisfacción, cómo perciben los turistas el destino, encontrando buenos resultados en general sobre la percepción de los beneficios obtenidos a partir de la imagen que se proyecta en la publicidad de Puerto Vallarta en los mercados, pero también mencionando los puntos de mejora en los que se debe trabajar en beneficio del destino (González, 2010). Este trabajo se realiza con base en la encuesta de salida de los turistas después de visitar el destino.

En la temática de Espacio, planeación, sustentabilidad y desarrollo local, en el subtema ordenamiento y planeación del espacio, se tienen diversos tipos de trabajos. El primero hace un análisis sobre los procesos de desarrollo en la región: usando 
el método Delphi (Fernández, 2009), se le consulta a expertos sobre los problemas para lograr convertirse en un lugar sustentable y sus posibles soluciones. Este texto pone en relieve la falta de un trabajo sistemático desde las esferas públicas, la escasa coordinación entre los agentes públicos y privados y la ausencia de acciones de largo impulso para mejorar las condiciones de la población que vive en la ciudad de Puerto Vallarta. En el mismo tenor se acentúa que existen las condiciones para que se realicen esfuerzos hacia revertir las condiciones negativas y potenciar las capacidades que tiene la ciudad, no sólo desde el turismo sino buscando alternativas productivas diferentes y complementarias a las condiciones del destino.

Se analizan las condiciones de planeación en el territorio de Puerto Vallarta y los conflictos en los espacios urbanos, de forma particular la tenencia de la propiedad y el uso del espacio turístico y habitacional que generan presión en una relación de convivencia y conflicto (López R., 2006); y la construcción de los valores de la propiedad de la tierra en el proceso de transformación de la ciudad turística (Tovar, 2006).

En el mismo sentido se analizan los procesos de conurbación entre Puerto Vallarta y Bahía de Banderas (Reyes, 2009), la importancia de contar con ordenamientos comunes en la planeación y el desarrollo urbano. Sobre este asunto, después de más de diez años, los gobiernos de ambos estados han iniciado los procesos jurídicos para hacer posible este tema que ha estado en la agenda pública y privada, toda vez que los criterios políticos frenan una realidad donde existe un solo destino en dos estados federales diferentes.

Un trabajo asociado a estos esfuerzos de conurbación tiene que ver con la propuesta de crear un sistema de transporte público marítimo que sea seguro y eficiente tanto para pobladores como visitantes, generando una descompresión en las vías terrestres, que por la orografía del territorio vuelve complicados los traslados a lo largo del destino, desde el centro de Puerto Vallarta a la marina, a la zona de nuevo Vallarta y a las localidades del litoral de Bahía de Banderas y Cabo Corrientes (Rendón, 2017).

En ese mismo sentido existe otra conurbación, aunque está en una pequeña localidad costera, donde por su configuración espacial la localidad de Boca de Tomatlán es parte de dos municipios (Puerto Vallarta y Cabo Corrientes), donde existe desatención por ambas autoridades, generando un proceso de presión urbana que se agrava por ser el principal puerto de embarcaciones locales que transitan por la vía marítima a las localidades del litoral del vecino municipio de Cabo Corrientes (Calzada, 2005).

La problemática del Centro Histórico es estudiada de forma importante. Se analiza la necesidad de una transformación urbana para regenerar la identidad de pueblo tradicional (Chavoya, 2006). También la nueva realidad de la zona Centro que ha 
perdido su capacidad de centralidad urbana, pero que es necesario replantear como tema puntual en la construcción de un producto asociado al destino (Jáuregui, 2018). Trasformación que debe sufrir el Centro y la ciudad, mejorando sus capacidades para que sea transitado por todas las personas. La ciudad no tiene un sistema de accesibilidad universal, no solo pensando en los turistas sino comprendiendo toda la población (local y visitantes), como un elemento indispensable de una ciudad sostenible (Dávalos, 2019).

Por la presión que se ejerce en las áreas marginales de Puerto Vallarta, se han realizado diversos trabajos que analizan la problemática urbana de las colonias asentadas allí, tomando como ejemplo a diversas localidades donde la marginación y la falta de servicios son la constante. Al identificar las características de la población, se desvela que en su mayoría son migrantes que se asientan en zonas provocando una presión en el medio natural, que las hacen insostenibles y con enormes carencias sociales (Baños, 2005; Tapia, 2006; Molina, 2017; Segura, 2017; García, 2019: Lara, 2019). Estos trabajos se han apoyado, en la mayoría de los casos, en instrumentos de análisis territorial que permiten identificar la situación de las comunidades.

En la temática de Sustentabilidad se han dividido los trabajos en cuatro apartados que valorizan las capacidades del espacio turístico para afrontar los retos de tener un destino sostenible. Los mecanismos de implementación del turismo en un territorio que originalmente fue agrícola y sufre transformaciones asociadas al turismo, ha generado amplias zonas de habitación de los trabajadores, de diversa calidad, generando impactos que afectan tanto al espacio como a las propias comunidades allí asentadas, que requieren atención.

La evaluación de las capacidades sostenibles es abordada en dos trabajos en el municipio de Bahía de Banderas (Castro, 2009) (Zepeda, 2019). En ambos trabajos se explora el espacio turístico a partir de la medición de indicadores sociales, encontrando convergencias en sus resultados. A pesar de la falta de información pertinente y actualizada para medir diversos indicadores, se explican las asimetrías sociales a pesar de las importantes capacidades económicas asociadas al turismo en la generación de empleos y en la creación de empresas. Se tienen problemas graves en el medioambiente por las graves afectaciones al territorio y al uso inadecuado del espacio, debido a la pésima planeación urbana que se refleja en la problemática social de las zonas de alta marginación.

Una de las problemáticas ambientales generadas por el desarrollo insostenible son los tiraderos de basura ilegales. Herrera (2019) analiza cómo los desechos provienen de las actividades económicas del turismo y de la población generando un pro- 
blema de contaminación, lo que se agrava por la falta de acciones desde la autoridad, al no tener mecanismos sostenibles para la recolección y confinamiento de desechos, no solo de basura, sino incluso de residuos peligrosos.

En un apartado realizado por profesionales de la biología, se evalúa la capacidad del territorio para soportar las actividades productivas en la región, particularmente el turismo y los impactos medioambientales que se ejercen sobre el territorio. A través de la medida de la huella ecológica (Chávez, 2007) se evalúa el impacto ejercido en espacios turísticos, demostrando el nivel de degradación y sus efectos, concluyendo que los visitantes tienen una mayor aportación en cuanto a la huella ecológica en el destino.

Puerto Vallarta está rodeado por espacios estuarinos. Se analiza la capacidad que tienen los manglares para soportar los impactos del turismo, demostrando el nivel de fragilidad que tienen y la importancia de su conservación, tanto por la estabilidad ambiental, como por ser un recurso usado por el turismo, por lo que se plantea su uso sostenible (Cruz, 2009).

Existen dos trabajos sobre especies animales emblemáticas de la región, la baIlena (Beets, 2006) y el cocodrilo (Hernández, 2008). En el caso de la ballena, las prácticas intensivas para el avistamiento en temporada (que va de diciembre a marzo) provocan conflictos y accidentes con los animales y entre las embarcaciones, por lo que es necesario hacer cumplir los ordenamientos legales y generar mecanismos para el cuidado de los lugares de paseo marítimo. En los cocodrilos, la expansión de los espacios habitacionales y turísticos ha afectado significativamente los hábitats naturales de esta especie protegida, generando conflictos y degradación. El esfuerzo de la Universidad de Guadalajara en la construcción de una unidad de manejo ambiental (UMA), encargada de la protección, atención y estudio de esta especie, es valorada en este trabajo, en el que se explica la importancia de mantener los esteros naturales con sistemas de protección para la vida de los cocodrilos y otras especies endémicas, fundamentales para el equilibrio natural de la región.

La importancia que tienen los arrecifes artificiales para el desarrollo de la pesca y el turismo sostenible en la costa de Jalisco debe ser reconocida, a fin de contribuir a mejorar las condiciones de la naturaleza y ampliar los esfuerzos en su conservación. Se considera indispensable incrementar los estudios sobre este tema (Kosonoy, 2008).

En la temática de Estudios de las comunidades locales, existen trabajos que muestran las transformaciones de las poblaciones asentadas en el territorio turístico. Por un lado, se da una transformación de sociedades agrícolas a sociedades que trabajan en los servicios turísticos, lo que ha provocado conflictos y cambios en las 
formas de vida de los habitantes que ven cambiar su estructura poblacional. Con el paso de las vías de comunicación se desarrollan actividades complementarias al turismo que integran a las comunidades a la novedosa actividad, dejando a un lado sus procesos tradicionales e involucrándose plenamente al turismo (Sánchez, 2005; Fernández, 2014).

Por otro lado, se evalúan las capacidades del territorio para la realización de actividades de turismo alternativo, explicando las bondades que tiene el espacio para el desarrollo de este tipo de proyectos. Las comunidades locales han usado los espacios de sus comunidades desarrollando proyectos que benefician a sus poblaciones con actividades de turismo alternativo de base comunitaria. Existen beneficios económicos y sociales, pero se tienen impactos en los espacios naturales por el número de visitantes y las limitadas acciones para prevenir estas situaciones (Ortiz, 2017). Además se avalúan las capacidades del territorio para la realización de proyectos ecoturísticos (Nájera, 2018).

Los proyectos de turismo alternativo que se ofertan de manera complementaria al turismo de masas deben mejorar sus capacidades. Al ser evaluados con indicadores de calidad se concluye que los proyectos analizados tienen buenas potencialidades para su operación, pero existen rezagos en los procesos de calidad empresarial, tanto en el servicio al cliente como en el cuidado al medio natural (Llamas, 2018).

Las sociedades también sufren impactos, cuando las comunidades que se han asentado son migrantes (Gómez, 2006) que llegan buscando mejoras de vida que les ofrece el turismo. Esta actividad absorbe mano de obra de forma intensiva y es imán para atraer grupos de migrantes, de forma preferente de zonas rurales empobrecidas del sur del país, pero también de profesionistas y técnicos de zonas urbanas. Una condición adicional es que las comunidades serranas, alejadas a las zonas turísticas de litoral, ven como una posibilidad integrarse al proceso de desarrollo turístico ofreciendo actividades complementarias como turismo rural sostenible (Rueda, 2005; Bravo, 2006).

Una comunidad que ha sido estudiada dos veces es Sayulita, una antes de que se declarara Pueblo Mágico (Villaseñor, 2010) y otra después de su denominación (Gutiérrez R., 2018). Esta comunidad sui generis de turismo de sol y playa, es analizada en el primer trabajo en la transición de un pueblo con actividades tradicionales a un pueblo turístico, que ha ido perdiendo tradiciones y en el que se implantan nuevas formas de vida. En el segundo se analiza el impacto que ha tenido como un espacio de moda y posteriormente nombrado pueblo mágico, lo que ha provocado un caos en los procesos urbanos, el incremento en los precios de las propiedades, el aumento en el 
costo de vida que afecta a la comunidad y que la mayor parte de los emprendimientos son de personas ajenas a la población. Lo más grave es el conflicto de inseguridad y la pérdida de la tranquilidad de los habitantes de la localidad.

La ampliación del turismo hacia la costa sur del estado de Jalisco ha provocado una ampliación de actividades productivas en los municipios vecinos de Cabo Corrientes (García, 2008) y Tomatlán (Noriega, 2013; Villanueva, 2019). Las transformaciones en la dinámica social y económica de las localidades de playa y serranos, y el incremento de los emprendimientos por sus atractivos naturales, los han convertido en espacios para el desarrollo de actividades turísticas. Un asunto que no ha permitido su pleno desarrollo es la problemática de la tenencia de la tierra en Cabo Corrientes, por su carácter indígena, lo que no permite la venta y transmisión de dominio. En Tomatlán la problemática se origina por los conflictos históricos en la propiedad de la tierra entre los ejidos y los pequeños propietarios.

\section{Discusión, aportaciones y conclusiones}

En los casi 20 años en la formación de investigadores desde el Posgrado en Ciencias para el Desarrollo, la Sustentabilidad y el Turismo, la experiencia ha sido enriquecedora tanto para quienes diseñaron esta idea y aportaron sus capacidades, como para quienes se han sumado en diversos momentos, teniendo como principales protagonistas a los estudiantes, quienes con su esfuerzo e ímpetu aportaron su trabajo.

Este trabajo demuestra el carácter multidisciplinar del programa, pues se basa en la búsqueda de soluciones a los problemas sociales con una visión integral. Se ha logrado gracias a las aportaciones de los profesores, quienes desde diversas disciplinas se han integrado al posgrado, situación nada fácil, pero sí muy gratificante a la luz de los resultados.

El modelo de desarrollo basado en el turismo es el principal enfoque que se encuentra en los trabajos que se presentan; la búsqueda del desarrollo como un logro de toda la sociedad es analizado desde diversas disciplinas, pero también de posturas personales. Sin que exista confrontación, se da un proceso de complementariedad. Se puede decir que existe unidad, desde la diversidad de explicaciones, que permiten entender de una mejor manera a la realidad, que es compleja, diversa y que requiere aproximaciones multidisciplinares para ser entendida.

Cada proyecto tiene a su vez un modelo metodológico particular, que se construye a partir de cada disciplina. En los trabajos se puede entender el nivel de desarrollo de los procesos para abordar el conocimiento, siendo cualitativos, cuantitativos o 
mixtos, con una amplia gama de instrumentos aplicados a la población local, a los expertos o a los prestadores de los servicios turísticos, haciendo comparación con otros territorios, con una permanente contrastación con las aportaciones teóricas en las que se basaron los trabajos realizados.

Los trabajos permiten configurar de una manera cercana cuál es la realidad de un destino turístico con enormes potencialidades, que, de seguir las tendencias actuales, se mantendrá como un referente nacional e internacional. Para ello, se deben considerar los retos planteados de regeneración del espacio con nuevos productos que lo revaloricen y manteniendo el liderazgo de los ya existentes, a partir de criterios de competitividad. Estas capacidades competitivas se deben dar a través de mecanismos responsables en el cuidado y protección de los íconos en los que basa su liderazgo, siendo los recursos naturales y el patrimonio cultural los pilares en los que se construye el destino.

De allí que los trabajos presentados dan cuenta de la fragilidad medio ambiental en la que se encuentra Puerto Vallarta, apuntando que deben construirse mecanismos de preservación y regeneración de forma urgente, cumpliendo con las disposiciones legales y mejorando criterios normativos a favor del medio ambiente, que implica estar a favor del propio destino. Se debe analizar la situación que guardan las condiciones identitarias y culturales, mismas que deben preservarse y usarse de la mejor manera, en favor de construir un destino sustentable.

La preocupación de los impactos del turismo en la sociedad es otro elemento de atención. Los grupos marginados que se instalan en zonas periféricas requieren trabajo y servicios públicos, asistenciales y educativos, pero estos requerimientos urgentes no se logran sin un proyecto de desarrollo de largo impulso. Se deben atender los efectos, pero se deben resolver ante todo las causas que les dieron origen.

En cuanto a procesos de planeación del desarrollo, se tiene la imperiosa necesidad de que se construyan mecanismos para su operación de forma sustentable. Que en el centro de toda acción se tenga como prioridad la protección del medio natural, la preservación de la cultura e identidad y la protección a los grupos sociales más vulnerables.

La realidad del destino es comprendida de una mejor manera al realizar un análisis a través de la honesta visión colectiva, que da cuenta de un mosaico diverso. Esto no se entiende si no es a partir de la comprensión de las partes, pero más aún, no se entienden las partes sin una comprensión del todo, que en este caso es el destino. Cada variable se relaciona de forma dialógica con el resto. Se comunican, pero también se enfrentan de forma dialéctica, donde los efectos se vuelven causa de forma 
recursiva. Comprender al destino Puerto Vallarta permite entenderlo como un sistema complejo y en transformación permanente.

Hacer un recuento de la actividad formativa de investigadores permite comprender la importancia que tienen los procesos científicos para describir de manera adecuada los problemas. Para plantear soluciones, se debe descubrir que existen diversos caminos para llegar a la comprensión de la realidad. Los enfoques son producto de reflexiones profundas, muchas veces encontrados, pero no por ello enfrentados. Más bien, son complementarios para tener una más amplia visión y no solo unidimensional. El trabajo académico en la formación de investigadores no concluye si estos no son capaces de generar mecanismos de vinculación con la sociedad, primero escuchando a todas las voces, para que con sus capacidades aporten soluciones que les sean significativas y que encuentren mecanismos para que las respuestas de largo plazo sean asumidas por todos los actores de forma responsable.

\section{Referencias}

Abreu-Hernández, L. F. y Cruz-Flores, G. (2015). Crisis en la calidad del posgrado Evaluación de la obviedad, o evaluación de procesos para impulsar la innovación en la sociedad del conocimiento. Perfiles educativos, XXXVII(147), 162-182.

Arnaiz, S., Pimentel, T. y Gauna, C. (2017). Educación y Turismo. México: Universidad de Guadalajara.

Baños, J. A. (2005). Asentamientos precarios en Puerto Vallarta del crecimiento informal al desarrollo urbano sustentable. El caso de las colonias "El progreso", "Lomas del progreso" y "12 de octubre" (tesis de maestría). Universidad de Guadalajara, Puerto Vallarta, Jalisco, México.

Barky, O., Dávila, M. (2016). El desarrollo de las carreras de posgrado en la Argentina: características generales, problemas recurrentes y desafíos. Revista de Educación superior, (2), 64-86.

Becerra, A. T. (2017). Alcances y limitaciones del posgrado en la Universidad Autónoma de Nayarit. Revista de la Educacion Superior, 46(183), 105-121. doi: https://doi.org/10.1016/j.resu.2017.05.003

Beets, K. D. (2006). Observación de ballenas en la Bahía de Banderas como actividad turística sustentable (tesis de maestría). Universidad de Guadalajara, Puerto Vallarta, Jalisco, México. 
Bravo, C. (2006). Turismo rural en el municipio de Bahia de Banderas, Nayarit: Una propuesta de desarrollo (tesis de maestría). Universidad de Guadalajara, Puerto Vallarta, Jalisco, México.

Bringas, N. L. y Verduzco, B. (2019). Política científica del turísmo en México. México: Academia Mexicana de Investigación Turística-Universidad de Guadalajara.

Cabral, J. (2006). Psicología y sociedad en una ciudad turística: Propuesta e intervención en problemáticas sociales sociales en una sociedad en transición, el caso Puerto Vallarta (tesis de doctorado). Universidad de Guadalajara, Puerto VaIlarta, Jalisco, México.

Cabrera, A. (2008). La gastronomía como elemento cultural para incorporar al turismo en el municipio de Cihuatlán, Jalisco (tesis de maestría). Puerto Vallarta: Universidad de Guadalajara.

Calderón, M. (2008). Evolución de la industria hotelera y su impacto económico en el desarrollo de Puerto Vallarta en la década de 1997- 2007 (tesis de maestría). Universidad de Guadalajara, Puerto Vallarta, Jalisco, México.

Calzada, M. T. (2005). "Boca de Tomatlán” Conurbación intermunicipal Estado de Jalisco (tesis de maestría). Universidad de Guadalajara, Puerto Vallarta, Jalisco, México.

Casas, C. A. (2017). Análisis de desigualdad social en Puerto Vallarta, Jalisco, México (tesis de maestría). Universidad de Guadalajara, Puerto Vallarta, Jalisco, México.

Castillo- Palacios, S. y Zúñiga-Collazos, A. (2015). Perspectivas de formación profesional en Colombia, enfocada en el sector turismo. Revista de investigación Universidad Quindo, 27, 98-108.

Castro, U. (2009). Estructuras regionales emergentes y desarrollo turístico sustentable: La región Costa Sur de Nayarit, México (tesis de doctorado). Universidad de Guadalajara, Puerto Vallarta, Jalisco, México.

Ceballos, L. A. (2009). Sociedad y religión en un municipio en transición. El caso de Bahía de Banderas, Nayarit (tesis de doctorado). Universidad de Guadalajara, Puerto Vallarta, Jalisco, México.

CEED-Cedestur (2001). Bahía de Banderas a futuro: construyendo el porvenir 20002025. México: Universidad de Guadalajara.

Celis, D. (2019). Análisis de la educación superior de turismo en America Latina y España. España: Editorial Síntesis- FITUR.

César, A. y Arnaiz, S. (2005). Turismo rural: modelos y propuestas. México: Universidad de Guadalajara. 
César, A. y César F. (2016). Educación universitaria y Turismo. Revista latinoamericana de turismología, 2(2), 8-17.

César, F. (2005). Ciudades turísticas: Desarrollo e imaginarios Careyes y Nuevo Vallarta (tesis de maestría). Universidad de Guadalajara, Puerto Vallarta, Jalisco, México.

Chávez, R. M. (2007). Huella ecológica y desarrollo sustentable. Un Enfoque Regional en la Costa Norte de Jalisco, México (tesis de doctorado). Universidad de Guadalajara, Puerto Vallarta, Jalisco, México.

Chavoya, J. I. (2006). El centro tradicional de Vallarta, rescate y reposicionamiento turístico (tesis de maestría). Universidad de Guadalajara, Puerto Vallarta, Jalisco, México.

Chavoya, M. L. (2013). Ser investigador: la zanahoria de los doctores recién egresados en México. Diálogos sobre Educación, 4(6), 1-16.

Conacyt (2019). Padrón Nacional de Posgrados de Calidad. Consejo Nacional de Ciencia y Tecnología. Recuperado de https://www.conacyt.gob.mx/index.php/ becas-y-posgrados/programa-nacional-de-posgrados-de-calidad

Contreras, O. y Urrutia, M. E. (2017). Trascendencia de un programa de posgrado en sus egresados. Revista Iberoamericana de Educación, 74(1), 59-74. https:// doi.org/10.35362/rie741626

Cornejo-Ortega, L., Chávez-Dagostino, R. y Espinoza-Sánchez, R. (2019). Prospectiva del turismo de naturaleza en la costa de Jalisco. Investigaciones Turísticas, (17), 189-212.

Cortés, P. A. (2008). El impacto del turismo electrónico (e-Tur) en la hotelería de Puerto Vallarta, México. La lucha entre las empresas trasnacionales y las locales (tesis de maestría). Universidad de Guadalajara, Puerto Vallarta, Jalisco, México.

Cruz, B. (2009). Diagnóstico ambiental y valoración de los recursos para fines turísticos de los ecosistemas de manglar en la Bahía de Banderas, México (tesis de maestríao). Universidad de Guadalajara, Puerto Vallarta, Jalisco, México.

Dávalos, A. Y. (2019). Plan municipal de accesabilidad: renovando la ciudad de Puerto Vallarta, hacia una ciudad humana e incluyente, en búsqueda de bienestar social y mayor competitividad. (tesis de doctorado). Universidad de Guadalajara, Puerto Vallarta, Jalisco, México.

DATATUR-Sectur. (2018). Compendio Estadístico del Turismo en México 2018. Recuperado de https://www.datatur.sectur.gob.mx/SitePages/CompendioEstadistico.aspx 
De Niz Sedano, A. G. (2018). Las empresas de alimentos y su inserción en el modelo del desarrollo local en la ciudad de Puerto Vallarta (tesis de maestría). Universidad de Guadalajara, Puerto Vallarta, Jalisco, México.

Fernández, C. (2014). El turismo como factor de transformacion del sector agrícola de Bahía de Banderas, Nayarit (tesis de doctorado). Universidad de Guadalajara, Puerto Vallarta, Jalisco, México.

Fernández, J. S. (2009). Prospectiva estratégica para el desarrrollo sustentable en Puerto Vallarta, utilizando el método Delphi (tesis de doctorado). Universidad de Guadalajara, Puerto Vallarta, Jalisco, México.

Franco, Y. (2006). Ordenamiento de la actividad turística de Puerto Vallarta Caso: Política Turística, propuesta de un marco orgánico funcional para el Ayuntamiento (tesis de maestría). Universidad de Guadalajara, Puerto Vallarta, Jalisco, México.

García, P. Y. (2019). Prácticas espaciales del migrante indígena en la ciudad turística. Un acercamiento al uso del espacio público en Puerto Vallarta, Jal. (tesis de maestría). Universidad de Guadalajara, Puerto Vallarta, Jalisco, México.

García, J. (2008). La perspectiva del desarrollo local y la importancia del turismo en el municipio de Cabo Corrientes Jalisco, México (tesis de maestría). Universidad de Guadalajara, Puerto Vallarta, Jalisco, México.

García, X. (2018). MIPYMES turísticas en Bahía de Banderas. Su incorporación a programas sustentables como estrategía de desarrollo (tesis de doctorado). Universidad de Guadalajara, Puerto Vallarta, Jalisco, México.

Gauna, C. y Vargas, R. (2017). Construcción de un posgrado de calidad en turismo, experiencia desde el posgrado en ciencias en ciencias para el desarrollo, la sustentabilidad y el turismo del CUCosta de la U. de G. En S. M. Arnaiz, T. Duarte y C. Gauna (coords.) Educación y Turismo (pp. 137-170). Puerto VaIlarta, Jalisco: Universidad de Guadalajara.

Gómez, A. B. (2006). Migración y turismo en el municipio de Bahía de Banderas. (tesis de maestría). Universidad de Guadalajara, Puerto Vallarta, Jalisco, México.

Gómez, S. (2018). La investigación del turismo y la formación de investigadores en México. México: Universidad de Guadalajara.

González, M. (2010). Análisis de la imagen proyectada y la satisfacción del turismo aéreo de Puerto Vallarta, Jalisco (tesis de maestría). Universidad de Guadalajara, Puerto Vallarta, Jalisco, México. 
González, N. (2006). El papel de los guías de turistas en un modelo de desarrollo sustentable. El caso de Puerto Vallarta, Jalisco (tesis de maestría). Universidad de Guadalajara, Puerto Vallarta, Jalisco, México.

Gutiérrez, N. (2018). Actitudes del residente ante el turismo sostenible. Adaptación y validación de una escala de medida (tesis de maestría). Universidad de Guadalajara, Puerto Vallarta, Jalisco, México.

Gutiérrez, P. (2018). Impacto del turismo en la calidad de vida del Pueblo Mágico de Sayulita: actores y participación local (tesis de maestría). Universidad de Guadalajara, Puerto Vallarta, Jalisco, México.

Hernández, P. (2008). La UMA reptiliario CIPACTLI una alternativa de desarrollo sustentable en Bahía de Banderas, México (tesis de maestría). Universidad de Guadalajara, Puerto Vallarta, Jalisco, México.

Hernández, L. y Nieto, J. (2010). La formación doctoral en México, historia y situación actual. Revista Digital Universitaria, 11(5), 1-9.

Hernández, V. (2010). El empleo en el turismo: ¿Flexible y sustentable? Análisis del uso de prácticas de flexibilidad laboral en la hotelería actual de Puerto Vallarta, Jalisco, México (tesis de maestría). Universidad de Guadalajara, Puerto VaIlarta, Jalisco, México.

Hernández, V. (2016). La marca de ciudad en la estrategia del desarrollo local: análisis de los imaginarios sociales y su afinidad con la marca turítica de Puerto Vallarta, Jalisco (tesis de doctorado). Universidad de Guadalajara, Puerto Vallarta, Jalisco, México.

Herrera, P. (2019). Tiraderos ilegales de residuos sólidos y su relación con la marginación en Puerto Vallarta, Jalisco (tesis de maestría). Universidad de Guadalajara, Puerto Vallarta, Jalisco, México.

Hiernaux, D. (2018). Más de tres décadas de investigación turística en México, algunas reflexiones. Dimensiones Turísticas, 2(2), 123-132.

Huerta, C. (2018). Condiciones de trabajo, funcionalidad familiar y apoyo social: estudio comparativo entre madres solas y madres de familias nucleares. (tesis de maestría). Universidad de Guadalajara, Puerto Vallarta, Jalisco, México.

Huizar, A. (2005). El impacto del turismo del segundo hogar en Puerto Vallarta. (tesis de maestría). Universidad de Guadalajara, Puerto Vallarta, Jalisco, México.

Instituto Nacional de Estadística y Geografía. (2010). Censo de población y vivienda 2010. Recuperado de https://www.inegi.org.mx/programas/ccpv/2010/ default.html 
Jáuregui, S. (2018). La centralidad histórica como referente de identidad urbana en la población local de Puerto Vallarta (tesis de maestría). Universidad de Guadalajara, Puerto Vallarta, Jalisco, México.

Jiménez, A. y Velázquez, M. (2017). Las universidades y el turismo. Reflexiones sobre la investigación turística en México. Dimensiones Turísticas, 1(1),143-156.

Kosonoy, D. (2008). Análisis del papel de los arrecifes artificiales para el desarrollo sustentable de la pesca y el turismo en la costa sur de Jalisco, Mexico (tesis de maestría). Universidad de Guadalajara, Puerto Vallarta, Jalisco, México.

Lara, K. (2019). Índice de prosperidad urbana en una ciudad turística, caso Puerto VaIlarta, Jalisco (tesis de maestría). Universidad de Guadalajara, Puerto Vallarta, Jalisco, México.

Llamas, J. (2018). Valoración del turismo alternativo mediante indicadores de sustentabildad en la Bahía de Banderas (tesis de maestría). Universidad de Guadalajara, Puerto Vallarta, Jalisco, México.

López, G. y Palomino, B. (2007). Elaboración de un diagnóstico del estado de la investigación turística en el país y generación de un programa integral de investigación para el sector turismo. Informe Técnico. Secretaría de Turismo, Distrito Federal, México. Recuperado de http://ru.iiec.unam.mx/1175/1/Diagnostico_Turistica.pdf

López, J. (2006). Territorio y turismo en Puerto Vallarta: Población y Perspectivas (tesis de maestría). Universidad de Guadalajara, Puerto Vallarta, Jalisco, México.

López, R. (2006). La artesanía del comercio ambulante como manifestación cultural hacia el turismo de Bucerías a Mezcales en Bahía de Banderas, Nayarit, México (tesis de maestría). Universidad de Guadalajara, Puerto Vallarta, Jalisco, México.

Marum; E. y Rosario, V. (2015). La calidad del pregrado y el posgrado, una mirada latinoamericana. México: Universidad de Guadalajara.

Molina, N. (2017). Identidades colectivas en colonias de origen informal en Puerto VaIlarta. (tesis de maestría). Universidad de Guadalajara, Puerto Vallarta, Jalisco, México.

Monterrubio, C. (2017). Necesidades de la investigación turística en México; reflexiones en el marco de la investigación internacional. Dimensiones Turísticas, 1(1), 31-52.

Mora, S. (2006). Turismo cultural en Puerto Vallarta (tesis de maestría). Universidad de Guadalajara, Puerto Vallarta, Jalisco, México. 
Nájera, A. (2018). Evaluación de la aptitud del territorio para turismo alternativo: caso corredor Miramar-Playa Tortugas, Riviera Nayarit (tesis de maestría). Universidad de Guadalajara, Puerto Vallarta, Jalisco, México.

Navarro, A. (2005). La empresa hotelera y el mercado de trabajo (Nuevo Vallarta, Bahía de Banderas, Nayarit) (tesis de maestría). Universidad de Guadalajara, Puerto Vallarta, Jalisco, México.

Noriega, E. (2013). Diseño del producto turístico competitivo: Caso Localidad José María Morelos, Tomatlán, Jalisco (tesis de maestría). Universidad de Guadalajara, Puerto Vallarta, Jalisco, México.

Núñez, P. (2008). Historia, turismo y territorio. Puerto Vallarta y su región, Bahía de Banderas (tesis de doctorado). Universidad de Guadalajara, Puerto Vallarta, Jalisco, México.

Núñez, S. (2008). La certificación en la empresa hotelera y el desarrollo sustentable. El caso de Puerto Vallarta, Jalisco, México (tesis de maestría). Universidad de Guadalajara, Puerto Vallarta, Jalisco, México.

Olivares, G. (2008). Problemas y Perspectivas para la Diversificación de la Oferta Turística y EI Desarrollo Sustentable en Puerto Vallarta, Jalisco (tesis de doctorado). Universidad de Guadalajara, Puerto Vallarta, Jalisco, México.

Ortiz-Villanueva, N. (2013). Calidad y posgrado en México, VII(3), 119-122.

Ortiz, D. (2017). Ecoturismo comunitario: calidad de vida, empoderamiento y conservación del ambiente (tesis de maestría). Universidad de Guadalajara, Puerto Vallarta, Jalisco, México.

Osorio, M.; Castillo, M. y Moreno, I. (2008). Investigación y conocimiento turístico en México. Un ecercamiento de las instituciones de educación superior y centros de investigación (IES y CIS). En Desarrollo turístico y sustentabilidad social (pp. 29-48). México: Miguel A. Porrúa.

Osorio, M. y Korstanje, M. (2017). Reflexiones en torno a la investigación turística. Dimensiones Turísticas, 1(1), 13-30.

Pacheco, T. (2011). La formación de doctores en educación en la UNAM (2000-2009). Revista Iberoamericana de Educación Superior, I/(4), 62-85. https://doi. org/10.22201/iisue.20072872e.2011.4.54

Pérez, S. P. (2018). Incertidumbre laboral y su relación con las alteraciones en la salud mental en personas que trabajan en la industria hotelera en Puerto Vallarta y Bahía de Banderas (tesis de maestría). Universidad de Guadalajara, Puerto Vallarta, Jalisco, México. 
Petroskova, B. (2019). Competitividad como forma del desarrollo: San Francisco, Nayarit (tesis de maestría). Universidad de Guadalajara, Puerto Vallarta, Jalisco, México.

Picazo, P., Moreno, S. y León, C. (2012). Difusión de la investigación científica de turismo en Brasil. CULTUR: Revista de Cultura e Turismo, 6(4), 4-36.

Pimentel, T., Costa, F. y Bifano, M. (2017). Distribución de la oferta educativa (OET) y las estructuras formales de investigación en turismo (EFIT) en Ecuador. Revista Turismo y sociedad, XXI, 193-215.

Pimentel, T. D. (2016). Distribución de la oferta educativa (OET) y las estructuras formales de investigación en turismo en México (EFIT) México, Revista Latino-Americana de Turismologia, 2(2), 25-48.

Pimentel, T., Carvalho, F. (2019). La sociología de la educación en turismo en Uruguay: un análisis de la oferta educativa en turismo y de sus centros de investigación. Revista Gestão Universitária na América Latina - GUAL, 12(3), 68-85. https://doi.org/10.5007/1983-4535.2019v12n3p68.

Rendón, H. J. (2017). Transporte y desarrollo sustentable: Una propuesta alternativa para Bahía de Banderas (tesis de doctorado). Universidad de Guadalajara, Puerto Vallarta, Jalisco, México.

Reyes, A. (2009). Análisis de la conurbación de la zona metropolitana interestatal de Puerto Vallarta- Bahía de Banderas (tesis de maestría). Universidad de Guadalajara, Puerto Vallarta, Jalisco, México.

Rivas, L. (2004). La formación de investigadores en México. Perfiles latinoamericanos: Revista de la Facultad Latinoamericana de Ciencias Sociales, Sede México, (25), 89-113.

Rodríguez, I. y Vargas, A. (2018). Aproximación reticular a la producción científica sobre turismo, realizada por investigadores mexicanos. Dimensiones Turísticas, 2(2), 9-26.

Rueda, O. F. (2005). Turismo rural alternativa de desarrollo sustentable en la zona cerrada de Bahía de Banderas, Nayarit; Mexico. (Comunidades El Colomo, El Coatante y Fortuna de Ballejo) (tesis de maestría). Universidad de Guadalajara, Puerto Vallarta, Jalisco, México.

Sánchez, F. E. M. (2005). "A los lados de la carretera" el desarrollo del turismo en Bucerías, Municipio de Bahía de Banderas, Estado de Nayarit (tesis de maestría). Universidad de Guadalajara, Puerto Vallarta, Jalisco, México.

Santos, I. A. (2017). Estimación del valor económico más verósimil del gasto en hospedaje en hotelería de 3 a 5 estrellas de Puerto Vallarta, tomando como año 
base 2015, mediante el uso de modelos determinísticos (tesis de doctorado). Universidad de Guadalajara, Puerto Vallarta, Jalisco, México.

Scartascini, G. (2009). Puerto Vallarta: La formación de un destino. Estudio de una sociedad en transición (tesis de doctorado). Universidad de Guadalajara, Puerto Vallarta, Jalisco, México.

Segura, J. R. (2017). Asentamientos humanos irregulares en el municipio de Puerto VaIlarta, Jalisco, México (tesis de maestría). Universidad de Guadalajara, Puerto Vallarta, Jalisco, México.

Silva, C. y Fransualdo, F. (2019). La relevancia del turismo como tema de investigación en el posgrado Strictu Sensue en Geografía en Brasil. Estudios y perspectivas del turismo, 28, 585-611.

Tapia, J. (2006). Análisis de los impactos por falta de planeación en asentamientos irregulares: caso colonia Ramblases del municipio de Puerto Vallarta, Jalisco, México (tesis de maestría). Universidad de Guadalajara, Puerto Vallarta, Jalisco, México.

Tiznado, E. (2007). Propuesta de un modelo de desarrollo sustentable y turismo de cruceros en Puerto Vallarta Jalisco (tesis de maestría). Universidad de Guadalajara, Puerto Vallarta, Jalisco, México.

Tovar, R. (2006). Turismo y la formación de los valores inmobiliarios. El caso de la de ciudad de Puerto Vallarta (tesis de maestría). Universidad de Guadalajara, Puerto Vallarta, Jalisco, México.

Vargas, E. (2013). Desarrollo, trabajo y empoderamiento en el contexto del turismo (Puerto Vallarta), desde un enfoque de género (tesis de doctorado). Universidad de Guadalajara, Puerto Vallarta, Jalisco, México.

Vargas, R. (2017). El emprendimiento como estrategia para propiciar el desarrollo local sostenible: caso Puerto Vallarta, Jal. (tesis de doctorado). Universidad de Guadalajara, Puerto Vallarta, Jalisco, México.

Villanueva, R. (2019). Turismo rural y factores que potencian el desarrollo local en el Valle de Tomatlán, Jalisco, Mexico (tesis de maestría). Universidad de Guadalajara, Puerto Vallarta, Jalisco, México.

Villaseñor, B. (2010). Sayulita un pueblo turístico. Experiencia de transición en el municipio de Bahía de Banderas, Nayarit (tesis de maestría). Universidad de Guadalajara, Puerto Vallarta, Jalisco, México.

Virgen, C. (2007). El desarrollo de un destino maduro: Puerto Vallarta, Jalisco, México (tesis de doctorado). Universidad de Guadalajara, Puerto Vallarta, Jalisco, México. 
Virgen, C. y Gauna, C. (2011). Información demográfica y económica de la región Puerto Vallarta- Bahía de Banderas: datos para la toma de decisiones. TURyDES Turismo y Desarrollo local, 4. Recuperado de: https://www.eumed.net/ rev/turydes/10/varl.htm

Virgen, C. (2014). Turismo y desarrollo sustentable. Un acercamiento al estudio del turismo. México: Ed. AMESTUR/U. de G./UFPR.

Zaldívar, M.; Canto, P. y Quintero, N. (2018). La calidad de los posgrados de formación docente en México. Publicaciones de la Facultad de Educación y Humanidades del Campus de Melilla, 48(1), 155-170. https://doi.org/10.30827/ publicaciones.v48i1.7333

Zepeda, A. (2018). Puerto Vallarta a través de sus imaginarios (tesis de doctorado). Universidad de Guadalajara, Puerto Vallarta, Jalisco, México.

Zepeda, S. (2019). Desarrollo y Turismo en el municipio de Bahía de Banderas, Nayarit (tesis de doctorado). Universidad de Guadalajara, Puerto Vallarta, Jalisco, México.

Zorrilla, M. (2006). Relación de la hotelería en el ciclo de vida de un destino turístico aproximaciones al caso de Vallarta (tesis de maestría). Universidad de Guadalajara, Puerto Vallarta, Jalisco, México. 